\title{
Tratamiento de un caso de trastorno límite de la personalidad en una comunidad terapéutica
}

RESUMEN: Se expone un caso clínico en el que coexisten un trastorno límite de la personalidad con un trastorno de la personalidad por evitación. Se describe el caso, la evaluación y el tratamiento combinado realizado durante un año de ingreso de la paciente en una Comunidad Terapéutica.

PALABRAS CLAVE: Trastorno limite de la personalidad, Comunidad Terapéutica.

\begin{abstract}
A clinic case is exposed in wich coexists a Borderline Personlaity Disorder and an Avoidant Personality Disorder. The case, the evaluation and the mix treatment during one year of hospitalization in a Therapeutic Comunity are described.
\end{abstract}

KEY WORDS: Borderline Personality Disorder, Therapeutic Comunity.

\section{Introducción}

El trastorno límite de la personalidad tiene como principal característica la intensidad y variabilidad de los estados de ánimo. Estas personas tienden a experimentar largos periodos de abatimiento y desilusión, interrumpidos ocasionalmente por breves episodios de irritabilidad, actos autodestructivos y cólera impulsiva. Estos estados de ánimo suelen ser imprevisibles y parecen ser desencadenados menos por sucesos externos que por factores internos (1).

En el DSM-IV (APA) este trastorno es definido como una pauta duradera de percepción, de relación y de pensamiento sobre el entorno y sobre sí mismo en la que hay problemas en diversas zonas: la conducta interpersonal, el estado de ánimo y la autoimagen. Según Beek et al (2) los rasgos que más impresionan son la intensidad de sus reacciones emocionales, la inestabilidad de sus estados de ánimo y la gran variabilidad de síntomas que presentan.

La primera publicación en la que se empleó la designación "limite" fue escrita por Adolf Stern en 1938. Este artículo surgió debido al creciente número de pacientes que no podían ser situados claramente en las categorías neurótica o psicótica y que, además, eran refractarios a la intervención psicoterapéutica. Este autor identificó 10 síntomas o rasgos de carácter definitorios de estos pacientes, y algunos de ellos se han mantenido y forman parte de los criterios de las concepciones contemporáneas del trastorno límite.

En un trabajo de revisión de tratamientos psicoterapéuticos en pacientes con trastornos limites de la personalidad, Diaz Curiel (3) concluye que el objetivo general 
del tratamiento psicoterapéutico es la disminución del riesgo de las conductas suicidas, los actings y la mejoría de los síntomas. Este autor plantea que determinar la terapia de elección no es fácil. Propone que en pacientes con escasa tolerancia a la frustración y un bajo umbral en el control de impulsos, está más indicado una terapia de grupo, la terapia interpersonal y las terapias conductuales comportamentales dirigidas hacia el control de los impulsos; el abordaje familiar es útil debido a la alta frecuencia de patología familiar. Igualmente, este autor mantiene que en muchas ocasiones, y debido a la gravedad de los síntomas, hay que pensar en un tratamiento mixto y/o combinado en el que participen tanto la farmacoterapia, como recursos sociales, hospitalizaciones de media o larga estancia, hospital de dia e incluso en algunos casos hay que tratarlos como casos crónicos y utilizar los recursos de rehabilitación disponibles.

La Asociación Americana de Psiquiatras (APA) es su Guía Práctica para el tratamiento de los pacientes con Trastornos de Personalidad Límite (4) propone, en base a la experiencia clínica, como tratamientos eficaces una combinación de psicoterapia individual (terapia psicoanalítica/psicodinámica y terapia dialéctica conductual), psicoterapia grupal, psicoeducación familiar y farmacoterapia.

La comorbilidad entre este trastorno y otros trastornos de personalidad es una cuestión ampliamente documentada (5). Millon (1) señala cómo las patologías estructurales o graves (entre ellos el trastorno límite de la personalidad) coexisten casi siempre con algún otro trastorno de la personalidad como el evitador. En este mismo sentido, la APA(4) plantea que la comorbilidad más frecuente suele ser con trastornos del ánimo, trastornos relacionados con abuso de sustancias y trastosnos alimentarios en el eje I; y con trastornos de personalidad antisocial, evitador e histriónico en el eje II.

El trastono de personalidad límite es el más frecuente de los trastornos de personalidad (30\%-60\% de la población con trastornos de la personalidad); representa el $10 \%$ de los sujetos vistos en consultas ambulatorias, entre el 15\%-20\% de los pacientes hospitalizados y supone el $2 \%$ de la población general (4).

Los pacientes con trastorno de la personalidad por evitación se aislan activamente, son hipersensibles a los estímulos sociales e hiperreactivos a los sentimientos y estados de ánimo ajenos, en especial a los que pueden sugerir rechazo y humillación. Su extrema ansiedad no sólo invade sus pensamientos e interfiere en sus comportamientos, sino que les distrae de objetivos placenteros, inclinándoles a distanciarse de los demás como mecanismo de protección contra el sufrimiento psíquico que anticipan.

Este trastorno se caracteriza por una evitación generalizada, conductual, emocional y cognitiva. Esa evitación se nutre de temas tales como la autodesaprobación, una expectativa de rechazo en las relaciones interpersonales y la creencia de que las emociones y los pensamientos desagradables no pueden tolerarse (2).

La etiqueta personalidad por evitación es nueva y fue acuñada por Millon en 1969 (1) como una designación descriptiva de los individuos que se distinguían por su aversión activa hacia las relaciones sociales, en contraposición a la aversión pasiva (ej. Personalidad esquizoide). 
INFORMES

En cuanto al tratamiento psicoterapéutico de este trastorno, Beck(2) propone en primer lugar establecer una alianza confiable entre terapeuta y paciente, encaminada a la identificación y modificación de pensamientos y creencias disfuncionales primero sobre esa relación terapéutica y que posteriormente le sirva para cuestionar sus creencias sobre otras relaciones. Plantea también este autor como aspectos fundamentales de la intervención: la posibilidad de situaciones de afrontamiento, los ensayos conductuales y el aprendizaje de Habilidades Sociales, y el aprendizaje de manejo de las emociones negativas.

\section{Exposición del caso}

Mujer de 30 años. La cuarta de 6 hermanos. Soltera, reside con sus padres, 4 hermanos, 1 sobrino y la abuela materna. Inactiva laboralmente. Percibe una pensión de la seguridad social.

Antecedentes personales:_Desde su primera infancia presenta problemas de relación con los demás; sólo hablaba (con monosílabos) con personas de su entorno familiar; en el colegio nulas relaciones con los compañeros. Presentaba conductas de evitación de las relaciones sociales "cuando entraba alguien de fuera a mi casa me escondía debajo de la cama”. Mantenía pensamientos de ser mala y querer morirse. Escaso rendimiento escolar.

A los 8 años pasaba largos ratos mirando al sol "porque quería quedarme ciega para no ver a la gente porque me daban miedo".

A los 11 años pensamientos de ahorcarse con fuertes deseos de muerte. Se veía distinta a las demás y pensaba que no era de este mundo, que era "extraterrestre".

A $\operatorname{los} 13$ años sufrió varios meses amenorrea estando convencida de estar embarazada de su padre, pues coincidió con el último embarazo de la madre quien le explicó que cuando una está embarazada no tiene la regla y que el bebé era de su marido, aplicándose para ella misma ambas explicaciones.

Estudios hasta $2^{\mathrm{a}}$ de BUP con escaso rendimiento. Abandona los estudios con 16 años comenzando a trabajar en una carpintería donde permanece un año y en la que sufre abuso sexual por parte del jefe, hecho que nunca denunció ni confesó a los padres. Ante este hecho reaccionó huyendo del domicilio familiar durante 12 dias. Fue encontrada por la policia en Sevilla, apareciendo mutista y asustada. El verdadero motivo no llegó a ser conocido por la policia ni por la familia.

Posteriormente trabajó durante otro año y medio en otra carpintería que abandonó por sugerirle los encargados que necesitaba atención psiquiátrica por presentar conductas de evitación, aislamiento y mutismo.

Situación familiar: Conviven en el domicilio familiar 9 miembros: ambos padres, 5 hermanos, un sobrino de 8 años (con retraso mental) y la abuela materna.

Padre: pensionista por accidente laboral. Bebedor. Ausente y desinteresado por los problemas familiares.

Madre: Diabética insulinodependiente y diagnosticada de T. ansioso-depresivo. 
Hermanos:

-Varón. Convive con los padres. Pensionista por invalidez. Bebedor.

-Varón. Vive en su propio domicilio. No se le conoce enfermedad mental.

-Mujer. Soltera, un hijo de 7 años. Diagnosticada de Déficit mental. El hijo presenta retraso en su desarrollo evolutivo.

- Varón. Soltero. Estudiante.

-Varón, 16 años. Conflictivo, rebelde, dificultades en acatar normas, fracaso escolar con varias expulsiones del centro. Supervisado por Tribunal de Menores por delito de daños a la propiedad.

Abuela: Sufre la enfermedad de Parkinson.

Historia de la enfermedad: La paciente consulta por primera vez en agosto de 1989, a los 17 años, tras sobreingesta medicamentosa con intención autolítica. Según la familia sufre intentos de suicidio desde los 14 años lo que ha motivado frecuentes consultas en Urgencias. Fue diagnosticada de T. Límite de la personalidad y Fobia social. Presentaba conductas de evitación y escape ante relaciones sociales, incapacidad de mirar a los ojos, convencimiento de que los demás se rien de ella porque es "mala". Consumo excesivo de alcohol, frecuentes conductas impulsivas auto y heteroagresivas, ánimo bajo, gran ansiedad. Homosexualidad egodistónica. Temporadas de anorexia restrictiva y otras con episodios bulímicos con vómitos autoprovocados.

A lo largo de la historia de su enfermedad ha tenido 4 ingresos en la Unidad de Agudos del H. Reina Sofía (en el 96, 97 (2), y 99) con tiempos de ingreso largos (3-4 meses). Las hospitalizaciones han sido causadas por intentos de suicidio mediante intoxicación medicamentosa y etílica, pensamientos obsesivos de "soy mala, me quiero morir" asociados a conductas autolesivas y conductas heteroagresivas con aparente descontrol de impulsos.

Ultimo ingreso en Unidad de Agudos desde Octubre del 99 hasta su traslado a la Comunidad Terapéutica en Febrero de 2000, durante éste realizó varios intentos de autolisis y presentaba pensamientos recurrentes de ser mala, deseos de muerte, tristeza, anorexia, intenso retraimiento social, importante agitación psicomotríz y acatisia en bipedestación y sentada. Desbordamiento familiar.

Juicio Clínico: -Trastorno de inestabilidad emocional de tipo Límite (F60.31. CIE-10)

-Trastorno ansioso (con conducta de evitación) de la personalidad (F60.6. CIE-10).

Tratamiento en Comunidad Terapeútica: Ingreso voluntario en Febrero de 2000.

Para la evaluación inicial de la paciente se utilizan las siguientes técnicas:

-Entrevistas clínicas individuales y familiares.

-Registros de observación de enfermería.

-Entrevista para valoración de psicoeducación.

-Entrevista dirigida para Habilidades Sociales (A. Blanco. Adapt. Arkowitz, H. 1981).

-Escala de Inteligencia de Wechsler para adultos. TEA. 1995.

Resultados de la evaluación inicial: 
INFORMES

1-Habilidades Funcionales: No presenta alteraciones en funciones cognitivas básicas como atención, concentración, memoria, aunque con frecuencia se vean mermadas por continuas rumiaciones. En cuanto a la capacidad intelectual los resultados del WAIS son los siguientes: CIV: 95; CIM: 83; CIT: 87. Se descarta la hipótesis de deficiencia mental que se ha barajado durante su historia personal pero que no se ha podido valorar hasta la actualidad por su mutismo mantenido. En cuanto a las habilidades de la vida diaria, presenta dificultades en manejo del dinero, uso de transportes públicos y habilidades domésticas por no haberlas adquirido tanto por su psicopatología como por la dinámica familiar. Presenta las siguientes conductas disruptivas:

-Auto y heteroagresiones.

-Deambulación rápida y continua por los pasillos.

-Tendencia al aislamiento y a meterse en cama ante situaciones que aumentan su ansiedad.

2-Autocuidado: Escaso nivel de autocuidado. Aseo personal inadecuado (no habito de aseo diario, no higiene bucodental). Dificultades en limpieza y cuidado del entorno (dormitorio, armario), así como del cuidado y lavado de su ropa. Dificultades en la masticación por ausencia de bastantes piezas dentales. Usa lentes correctivas por importante pérdida de visión. Se queja de dolor de espalda por alteración postural continua del cuello, inclinado totalmente hacia el pecho para evitar el contacto visual.

3-Conciencia de enfermedad: Aceptación de la enfermedad mental pero escaso conocimiento de la misma. Para la paciente, diversos síntomas de su Trastorno de Personalidad por Evitación son pruebas de su maldad ("soy mala porque no tengo amigos, no puedo hablar con la gente"). Niega posibilidad de mejoría pues los síntomas serían características intrínsecas de ella misma que no pueden ser modificadas. Conoce tratamientos farmacológicos, aunque no efectos principales ni secundarios. No autonomía en la toma de medicación por riesgo de sobreingesta.

4-Habilidades Sociales: La paciente presenta importantísimos déficits en esta área. Presenta incapacidad total de mantener contacto visual y grandes dificultades en HHSS (Habilidades Sociales) básicas (escuchar, iniciar conversaciones,etc.) En HHSS complejas (responder al fracaso, planificación, resolución de problemas,etc.) la incapacidad es total.

5-Apoyo Social: Nulo fuera del entorno familiar. En cuanto al apoyo familiar es escaso e inadecuado por el alto nivel de psicopatología en la propia familia. Dinámica familiar muy patológica que tiende a bloquear y a impedir la independización y autonomía de sus miembros. Situación de continua rivalidad, celos y conductas manipulativas de los hijos para acaparar la atención y cuidado de la madre.

6-Psicopatología: La paciente presenta la siguiente sintomatología:

-Frecuentes conductas auto y heteroagresivas. Golpea con los puños a pacientes y personal, justificándolo por aumentos bruscos de la ansiedad " se ríen de mí por dentro porque soy mala”. Se golpea la cabeza contra la pared para castigarse por su maldad. Varios intentos de autolisis con deseos de muerte (intento de ahorcamiento, ingesta de baterias de radiocassetts, prender fuego a su cama). 
-Estados de ánimo muy intensos y variables desencadenados más por factores internos que por sucesos externos. Sentimientos crónicos de vacío y soledad.

-Pensamientos estructurados de forma dicotómica y esquemas de pensamiento distorsionado ("tengo dificultades en las relaciones sociales, por lo que soy mala y me tengo que castigar y los demás también”). Estos pensamientos aunque son repetitivos, invasivos y que la paciente vive como que no puede controlar, no son vividos como absurdos, por lo cual los calificamos de ideas sobrevaloradas. La certeza de estos pensamientos fluctúa en distintos momentos. Mantiene que todo lo negativo que ocurre a su alrededor es culpa suya, lo que reafirma su pensamiento de ser mala y su necesidad de castigarse (Ej: alcoholismo de familiares, embarazo de su hermana..), mientras niega la responsabilidad sobre sus actos agresivos argumentando que son impulsos que no puede controlar, no sintiendo arrepentimiento ni culpa.

-Presenta una excesiva dependencia de la figura materna con una necesidad y deseos continuos de cuidado protector, atención y afecto. Celos y conductas manipulativas para atraer sus preocupaciones y cuidados. Temor al abandono y a la pérdida del cuidado de la madre. La familia presenta unos esquemas aprendidos, mantenidos y compartidos por todos sus miembros según los cuales todo lo que viene de fuera puede ser peligroso y destructivo para la unidad familiar, imponiendo un código de conducta según el cual no deben hacer partícipes a nadie de fuera (ni siquiera al terapeuta) de los conflictos, problemas o conductas de los miembros de la familia (lo que no nos ha permitido obtener información sobre sospechas de malos tratos y abusos sexuales). Es una familia aglutinada con gran influencia y repercusión emocional entre los miembros. Todo esto sitúa a la paciente en una continua lucha y ambivalencia dentro de las siguientes polos: dependencia/independencia; situación familiar negativa/mundo exterior peligroso; necesidad de expresar/prohibición de hacerlo. La imposibilidad de expresar hace que comunique sus sentimientos, deseos, temores mediante actos impulsivos, agresivos y manipulativos.

-Evitación y escape de situaciones sociales. Intolerancia de estar rodeada de varias personas.

-Relaciones interpersonales intensas y cambiantes pasando de una idealización a una devaluación (con compañeros, personal y, por supuesto, con la terapeuta), propias de sus esquemas cognitivos dicotómicos.

-Episodios de abusos importantes de alcohol con intención autolítica o como facilitador de las relaciones sociales al disminuir la ansiedad.

-Episodios de alteraciones de la conducta alimentaria con restricción de la ingesta y vómitos autoprovocados.

-Homosexualidad egodistónica. Para la paciente su homosexualidad es una prueba más de su maldad.

Intervenciones terapéuticas:

-Psicoterapia individual: Al inicio, la intervención se centró en establecer una 
INFORMES

relación terapéutica cooperativa; ésto implica un modo de trabajo en el que se evitan la lucha de poder y el excesivo control y directividad, buscando continuamente el consenso en los objetivos y las estrategias con el objetivo de lograr la confianza de la paciente superando el conflicto entre el deseo de ayuda y aceptación por un lado y el miedo a la vulnerabilidad y el rechazo por otro. Para ello se tratan explícitamente las dificultades de la paciente para confiar en el terapeuta. Se abordan de manera rápida y directa las respuestas emocionales fuertes. Durante el proceso de psicoterapia se intenta mantener un equilibrio en las sesiones entre un abordaje metódico y estratégico focalizado, y las preocupaciones inmediatas de la paciente; buscando bajo cada preocupación, problema o crisis concreta, los esquemas cognitivos o estrategias conductuales subyacentes. Las intervenciones iniciales se centran en objetivos conductuales concretos, trabajando con las conductas auto y heteroagresivas y con las respuestas emocionales intensas intentando que la paciente las reconvierta en contenidos y expresiones verbales. En este sentido, es también fundamental trabajar el miedo al cambio y las conductas de autoperpetuación. Para ir propiciando el control sobre las emociones, fue fundamental la reducción del pensamiento dicotómico. El control de los impulsos se trabajó explorando las motivaciones, pensamientos y sentimientos, y buscando alternativas de conducta favorables. El trabajo con técnicas cognitivas va facilitando el dasarrollo de una identidad más clara, ayudándola a detectar sus características y logros positivos y ayudándola a evaluar con realismo sus propias acciones. La psicoterapia individual se llevó a cabo en sesiones de 45 minutos con una frecuencia de 3 o 4 a la semana según el momento.

-Psicoterapia grupal: Debido a las dificultades de la paciente en establecer, mantener e incluso tolerar relaciones sociales mínimas, su inclusión en actividades grupales se hizo de forma paulatina y dejando siempre a decisión de la paciente el entrar, permanecer, y el grado de participación en las mismas. En sesiones individuales se analizaba y consensuaba con la paciente las actividades grupales a las que se iba incorporando, trabajando los pensamientos y emociones que despertaban. Una vez acordada su inclusión en la actividad, cada evitación o escape era analizada y modificados los aspectos cognitivos necesarios para ir consiguiendo mayor tiempo, implicación y participación en los grupos. Se comenzó incluyéndola en actividades grupales como juegos de salón, grupo de lectura de prensa; paulatinamente (tres meses después )se fue incorporando a los siguientes grupos terapéuticos: Psicoeducación, Educación para la salud, Entrenamiento en Habilidades Sociales y Grupo Terapéutico. Por último se incorporó a actividades grupales fuera de la Unidad (salidas culturales, asistencia a un polideportivo, senderismo..) al año de su ingreso.

-Psicoterapia familiar: Con la familia se trabajó los siguientes aspectos:

-Un proceso de psicoeducación familiar sobre la enfermedad de la paciente, sus pensamientos, sentimientos y conductas; de tal forma que la familia llegara a comprender estos aspectos de la paciente. 
-Evaluación y modificación de las pautas de interacción familiar.

-Evaluación y modificación de los esquemas cognitivos negativos compartidos por la familia. familia.

-Propiciar un mayor grado de autonomía de la paciente con respecto a su

Las sesiones familiares comenzaron al mes del ingreso. Se realizaron semanalmente con una duración de 60 minutos durante los cuatro primeros meses. Luego se mantuvieron sesiones quincenales durante dos meses y por último sesiones mensuales durante seis meses. A pesar de estar invitados todos los miembros de la familia, acudieron siempre los padres y algún hermano lo hizo de forma poco estable.

-Terapia ambiental: trabajar con la paciente dentro de la Comunidad Terapéutica nos permite utilizar la terapia a través del "medio" como otra estrategia terapéutica. En este sentido, la C.T nos proporciona tres aspectos importantísimos por las características de esta paciente:

- Proporcionarle un ambiente organizado y con un alto poder de control externo. Se le proporciona así una seguridad a partir de la cual poder trabajar. En este sentido es de suma importancia la coherencia y coexión del equipo terapéutico.

- Proporcionarle oportunidades de interacciones sociales con alta frecuencia y variabilidad. Esto permite obtener gran cantidad de material utilizable en la terapia individual y grupal.

- Proporcionarle oportunidades de aprendizaje social continuas.

-Farmacoterapia: A su ingreso se instauró tratamiento con Olanzapina y Loracepam a dosis altas, además de Valproato. Posteriormente se asoció Clomipramina y Trazodona y se sustituyó Olanzapina por Levomepromacina. Se controló el insomnio con Flunitrazepam. Más adelante se modificó Loracepam por una Benzodiacepina de acción larga (Cloracepato). Ultimamente se ha introducido en el tratamiento Topiramato con resultados positivos en el control de los impulsos.

Evolución: Tras un año de estancia y tratamiento en la Comunidad Terapéutica la situación de la paciente es la siguiente:

-Hay una mejora importante en aquellas conductas que se consideraron más de riesgo o disruptivas y en las que se centraron como objetivos las intervenciones iniciales. Aparece una disminución progresiva en las conductas auto y heteroagresivas, pasando de un total de 19 en el mes de Marzo de 00 (15 leves y 4 graves o de riesgo vital) a 1 leve en febrero de 01 (Gráfico 1). La conducta de deambulación rápida por la unidad disminuye progresivamente en frecuencia y duración hasta desaparecer totalmente. Disminuye, así mismo, la tendencia a aislarse en su dormitorio, pasando la mayor parte del dia en el salón común.

-Se produce una mayor tolerancia a las relaciones sociales, llegando a participar activamente en las actividades grupales tanto terapéuticas como de ocio y desapareciendo 
INFORMES

prácticamente las conductas de evitación y escape.

-Como resultado del afrontamiento continuo de situaciones sociales dismiye el miedo a las mismas y paralelamente van aumentando sus capacidades y deseos de comunicación verbal con las personas de su entorno (influyendo en la disminución de sus conductas de acting-out). Llega a expresar tanto a su familia como en los grupos terapéuticos, el abuso sexual sufrido en el trabajo y su homosexualidad.

-Mayor estabilidad emocional con disminución de las conductas impulsivas y las emociones extremas, producto también, de un cambio en el pensamiento dicotómico.

-Se produce una disminución progresiva de la dependencia con la familia, pasando de visitas prácticamente diarias de los padres provocadas por conductas manipulativas de la paciente para conseguirlo, a tolerar ver a la familia cada 15 dias (coincidiendo, con frecuencia, con permisos de fin de semana de la paciente en casa). Se va consiguiendo, también, un mayor conocimiento familiar de la paciente y sus problemas; propiciando todo ello una retroalimentación sobre la paciente y su comportamiento en los permisos más adecuada y útil.

-La paciente posee una mayor información sobre su enfermedad y aquellos aspectos que pueden ser modificados y cómo; esto aumenta su motivación para el control de la ingesta alcohólica y las conductas alimentarias problemáticas.

-Por último, se produce una adecuación y normalización importantes en los hábitos de autocuidado y de cuidado de su entorno.

\section{Comentarios.-}

Una de las cuestiones a decidir en la valoración inicial de los pacientes con trastorno de personalidad limite, es el marco o contexto de tratamiento; la APA (4), propone tres niveles de cuidados con indicaciones específicas: hospitalización parcial, hospitalización breve y hospitalización prolongada. Las indicaciones para esta última incluyen: riesgo de suicidio y conductas autodestructivas severas, presencia de un trastorno de eje I resistente y que supone un riesgo potencial para la vida (trastorno alimentario o trastorno del ánimo), presencia de abuso de sustancia resistente al tratamiento ambulatorio o de hospitalización parcial, continuo riesgo de agresiones a terceros, y síntomas con la gravedad suficiente como para interferir en su funcionamiento laboral, familiar y que no responden a otro marco de tratamiento.

Maxwell Jones definió en 1962 "una comunidad terapéutica distinguiéndola de otros centros de tratamiento por la forma en que el total de los recursos de la institución, tanto equipo como pacientes, se abocan autoconscientemente a la tarea de llevar adelante el tratamiento" (6).

Aunque no existe un modelo claro de comunidad terapéutica, Clark (6) establece ciertas características que se han observado en la mayoría de ellas:

1-Libertad para comunicarse: esfuerzo constante para abrir canales de comunicación y liberar los bloqueos.

2-Análisis de todos los sucesos en la comunidad terapéutica en función de la dinámica individual e interpersonal. 
3-Provisión de experiencias de aprendizaje.

4-Achatamiento de la pirámide de autoridad. La comunicación abierta elimina muchos de los estratos jerárquicos que dificultan el trabajo en otro tipo de unidades.

5-Examen de rol. El examen de lo que se hace, el por qué y cómo afecta a otros, lleva a una continua evaluación y modificación de la manera de trabajar. Esto puede conllevar cierto desdibujamiento de roles.

6-Reunión de comunidad. Es el lugar fundamental de debate de todos los procesos antes comentados. Es la característica fundamental de la comunidad terapéutica.

Creemos que unidades de este tipo pueden ofrecer un tratamiento adecuado a personas con este tipo de trastornos por varios motivos:

-Proveer un ambiente de contención tan necesario, teniendo en cuenta que el Trastorno Límite de la Personalidad tiene una incidencia de suicidio del 9\%, comparable con el riesgo de suicidio en la Esquizofrenia (7).

-La oportunidad de un tratamiento combinado, multiprofesional y de larga duración; cómo mínimo un año de tratamiento parece necesario en estos cuadros (4) (7).

Si pensamos en la cuestión costo-beneficio de este tipo de intervenciones, no podemos olvidar que este tipo de pacientes hacen un gasto excesivo de recursos de urgencias y de hospitalizaciones en unidades de agudos.

Para finalizar, comentar que nuestra paciente continúa, tras dos años, en la comunidad terapéutica y su evolución sigue siendo positiva aunque irregular. Su sintomatología continuamente cambiante y con reagudizaciones frecuentes, y los interminables conflictos que provoca en la comunidad, supone un continuo reto para todos nosotros. 
INFORMES

\section{BIBLIOGRAFIA}

(1) Millon T., Trastornos de la personalidad. Más allá del DSM-IV, Barcelona, Masson, 1998.

(2) Beck A.T., Arthur Freeman y otros, Terapia cognitiva de los trastornos de personalidad, Barcelona, Paidos, 1995.

(3) Diaz Curiel J., "Revisión de tratamientos psicoterapéuticos en pacientes con trastornos borderline de personalidad", Revista de la Asociación Española de Neuropsiquiatría, 2001, vol XXI, na 78, pp 51-70.

(4) American Psychiatric Association(APA), "Practice Guideline for the Treatment of Patinets with Borderline Personality Disorder", The American Journal of Psychiatry, 2001, vol $158, n^{\mathrm{a}} 10$, (suplemento).

(5) Becker D.F. et al, "Comorbidity of borderline personality disorder with other personality disorders in hospitalized adolescent and adults", The American Journal of Psychiatry, 2000, vol 157, na 12, pp 2011-2017.

(6) Clark, D.H. Psiquiatría administrativa. Los roles en la comunidad terapéutica, Buenos Aires, Ediciones Nueva Visión, 1973.

(7) Kraft Goin M., "Practical Psychotherapy: Borderline personality disorder: the importance of establishing a treatment framework", Psychiatric Services, 2001, vol 52, n² 2, pp 167-169.

*Psicóloga Clínica. Comunidad Terapéutica, Hospital Universitario Reina Sofia. Córdoba.

Correspondencia: Inmaculada Carranza Almansa. C/ Cantueso, Nª 24, 14012, Córdoba.

Fecha de recepción: 22-02-02 